\title{
Ambulatory (AASI), but not home (HASI), arterial stiffness index is associated with aortic pulse wave velocity
}

Hypertension Research (2011) 34, 402-403; doi:10.1038/hr.2010.248; published online 16 December 2010

The dynamic relation between systolic and diastolic blood pressure (BP) in 24-h ambulatory BP recordings reflects arterial stiffness. ${ }^{1,2}$ We defined the ambulatory arterial stiffness index (AASI) as unity minus the regression slope of diastolic on systolic $\mathrm{BP}$ in 24-h BP recordings. AASI correlated with aortic pulse wave velocity (aPWV) and the central and peripheral pulse pressures and augmentation indexes. ${ }^{1}$ Furthermore, independent of 24-h pulse pressure and aPWV, AASI predicts cardiovascular mortality and morbidity, in particular fatal and nonfatal stroke. ${ }^{2,3}$ AASI, therefore, holds promise for the stratification of cardiovascular risk.

In this journal, Stergiou et al. ${ }^{4}$ recently introduced the home arterial stiffness index (HASI) based on the self-measured BP. They reported that HASI correlated less with age, systolic BP and pulse pressure than AASI. They proposed that HASI might not replace AASI in the assessment of arterial stiffness. However, the study by Stergiou et al. ${ }^{4}$ did not include a direct measure of arterial stiffness. We, therefore, compared the association of aPWV with HASI and AASI in 67 consecutive patients, all untreated, who were referred for the diagnosis of hypertension to Ruijin Hospital. Within 1 week, the patients underwent 24-h ambulatory BP monitoring, selfmeasurement of BP at home, and an assessment of aPWV. Oscillometric SpaceLabs 90217 monitors (SpaceLabs Inc, Redmond, WA, USA) recorded BP at 20-min intervals from 0800 to $2200 \mathrm{~h}$, and at 30 -min intervals from 2200 to $0800 \mathrm{~h}$. Daytime and nighttime ranged from 0800 to $1800 \mathrm{~h}$ and from 2300 to $0500 \mathrm{~h}$, respectively. Patients measured BP at home in the morning (before breakfast) and in the evening ( $2 \mathrm{~h}$ after dinner) on 7 consecutive days, using the Omron HEM-7051 device (Omron Health Care, Kyoto, Japan).
Each self-measurement session consisted of three readings at 1 -min intervals after subjects had relaxed for $5 \mathrm{~min}$ in the sitting position. aPWV was measured using a high-fidelity SPC-301 micromanometer (Millar Instruments, Houston, TX, USA) interfaced with a laptop computer running the SphygmoCor software, version 8.2 (AtCor Medical, New South Wales, Australia). We compared means and correlation coefficients, using two-tailed tests for paired observations with the 24-h ambulatory statistics as reference. Bonferroni's correction for multiple comparisons was applied.

Of 67 participants, $34(50.8 \%)$ were female and $41(61.2 \%)$ had ambulatory hypertension (24-h BP $\geqslant 130 / 80 \mathrm{~mm} \mathrm{Hg}$ ). Age averaged 53.6 years (range, 30-73) and aPWV 8.0 \pm $1.6 \mathrm{~m} \mathrm{~s}^{-1}$. The number of BP readings was highest for 24-h AASI (Table 1). HASI was higher $(P<0.001)$ than 24 -h and nighttime AASI, but similar to daytime AASI $(P=0.20$

after Bonferroni's correction). The 24-h AASI $(r=0.43 ; P=0.003)$, but not daytime AASI $(r=0.11 ; P=0.36)$ and nighttime AASI $(r=$ $0.22 ; P=0.07)$, significantly correlated with aPWV. The significant correlation of 24-h AASI with aPWV was of similar magnitude as in our previous study (correlation coefficient, $0.51 ; P=0.49$ for unpaired comparison). ${ }^{1}$ aPWV did not significantly correlate with any estimate of HASI, irrespective of the timing or number of BP self-measurements $(P \geqslant 0.28$, Table 1$)$. In pairwise comparisons, correlations of aPWV with HASI were significantly weaker than those with 24-AASI (Table 1). These findings contrast with those of Qureshi et al., ${ }^{5}$ who reported that in 21 subjects aPWV correlated with HASI $(r=$ $0.45 ; P=0.04 ; P=0.064$ for unpaired comparison with our current estimate of -0.01 ).

In conclusion, aPWV correlates closer with AASI than HASI. In keeping with the suggestion Stergiou's of et al., ${ }^{4}$ for the derivation of

Table $1 \mathrm{AASI}$ and HASI in relation to aPWV in 67 untreated patients

\begin{tabular}{|c|c|c|c|c|}
\hline \multirow[b]{2}{*}{$\begin{array}{l}\text { Stiffness } \\
\text { index }\end{array}$} & \multirow[b]{2}{*}{$\begin{array}{l}\text { Mean number of } \\
B P \text { readings } \pm \text { s.d. }\end{array}$} & \multirow[b]{2}{*}{$\begin{array}{c}\text { Mean stiffness } \\
\text { index } \pm \text { s.d. }\end{array}$} & \multicolumn{2}{|c|}{$a P W V$} \\
\hline & & & $r$ & $P$ \\
\hline \multicolumn{5}{|l|}{$A A S I$} \\
\hline 24-h & $59.0 \pm 5.8$ & $0.42 \pm 0.11$ & 0.43 & 0.003 \\
\hline Daytime & $26.9 \pm 4.0^{\ddagger}$ & $0.53 \pm 0.17^{\ddagger}$ & $0.11^{*}$ & 0.36 \\
\hline Nighttime & $11.8 \pm 0.5^{\ddagger}$ & $0.42 \pm 0.21$ & 0.22 & 0.07 \\
\hline \multicolumn{5}{|l|}{ HASI } \\
\hline All & $39.4 \pm 6.7 \ddagger$ & $0.58 \pm 0.16 \ddagger$ & $-0.01^{\dagger}$ & 0.96 \\
\hline Morning & $20.2 \pm 2.8^{\ddagger}$ & $0.67 \pm 0.20^{\ddagger}$ & $0.13^{*}$ & 0.28 \\
\hline Evening & $19.2 \pm 4.1^{\ddagger}$ & $0.57 \pm 0.19^{\ddagger}$ & $0.08^{*}$ & 0.54 \\
\hline
\end{tabular}

Abbreviations: AASI, ambulatory arterial stiffness index; aPWV, aortic pulse wave velocity; BP, blood pressure; HASI, home arterial stiffness index.

Significance of the difference vs. 24-h AASI after Bonferroni's correction for multiple comparisons $(n=5):{ }^{*} P<0.05$; ${ }^{\dagger} P<0.01$; and $\ddagger P<0.001$. 
the arterial stiffness index, BP self-measurement should not be considered as alternative to 24-h ambulatory monitoring. As already reported before, ${ }^{6}$ recording BP for $<24 \mathrm{~h}$ can also not be recommended. Furthermore, AASI is determined by the ratio of the number of night to day BP readings. ${ }^{7}$ When the time intervals between $\mathrm{BP}$ measurements were longer during the nighttime, as in our study, the influence of nocturnal BP characteristics on the derived AASI values might be underestimated. It would be reasonable that for the determination of AASI, time intervals between successive BP readings were equal during the daytime and nighttime periods.

Ting-Yan $\mathrm{Xu}^{1}$, Yan $\mathrm{Li}^{1}$, Wang-Xiang $\mathrm{Fan}^{1}$, Fa-Hong Li ${ }^{1}$, Jun Zou ${ }^{1}$, Ping-Jin Gao ${ }^{1}$, Jan A Staessen ${ }^{2,3}$ and Ji-Guang Wang ${ }^{1}$
${ }^{1}$ Centre for Vascular Evaluations, Shanghai Institute of Hypertension, Ruijin Hospital, Shanghai Jiaotong University Medical School, Shanghai, China; ${ }^{2}$ Studies Coordinating Centre, Division of Hypertension and Cardiovascular Rehabilitation, Department of Cardiovascular Diseases, University of Leuven, Leuven, Belgium and 3 Department of Epidemiology, Maastricht University, Maastricht, The Netherlands E-mail: liyanshon@yahoo.com

1 Li Y, Wang JG, Dolan E, Gao PJ, Guo HF, Nawrot T, Stanton AV, Zhu DL, O'Brien E, Staessen JA. Ambulatory arterial stiffness index derived from 24-h ambularoty blood pressure monitoring. Hypertension 2006; 47 359-364.
2 Dolan E, Thijs L, Li Y, Atkins N, McCormack P, McClory S, O'Brien E, Staessen JA, Stanton AV. Ambulatory arterial stiffness index as a predictor of cardiovascular mortality in the Dublin Outcome Study. Hypertension 2006; 47: 365-370.

3 Hansen TW, Li Y, Staessen JA, Jeppesen J, Rasmussen S, Wang JG, Thijs L, Ibsen H, Safar ME, Torp-Pedersen C. Independent prognostic value of the ambulatory arterial stiffness index and aortic pulse wave velocity in a general population. J Hum Hypertens 2008; 22: 214-216.

4 Stergiou GS, Kollias A, Rarra VC, Nasothimiou EG, Roussias LG. Arterial stiffness index based on home (HASI) vs ambulatory (AASI) blood pressure measurements. Hypertens Res 2010; 33: 731-736.

5 Qureshi G, Lazar JM, Javaid H, Weber M, Salciccioli L. Self-measured (blood pressure) arterial stiffness index: a promising new measure of arterial stiffness. J Am Soc Hypertens 2007; 1: 347-352.

$6 \mathrm{Li} \mathrm{Y,} \mathrm{Hansen} \mathrm{TW,} \mathrm{Staessen} \mathrm{JA.} \mathrm{More} \mathrm{information}$ on the reproducibility of the ambulatory arterial stiffness index. Am J Hypertension 2010; 23: 113-114.

7 Dechering DG, Adiyaman A, van der Steen M, Thien T. Interstudy variability in the ambulatory arterial stiffness index. Hypertension 2007; 50: e65. 